\title{
Covalent modification of highly ordered pyrolytic graphite with a stable organic free radical using diazonium chemistry
}

\author{
Gonca Seber, ${ }^{\mathrm{a}}$ Alexander V. Rudnev, ${ }^{\mathrm{b}}$ Andrea Droghetti, ${ }^{\mathrm{c}}$ Ivan Rungger, ${ }^{\mathrm{d}}$ Jaume Veciana, ${ }^{\mathrm{a}}$ Marta \\ Mas-Torrent, ${ }^{a}$ Concepció Rovira, ${ }^{a}$ Núria Crivillers ${ }^{a}$
}

\begin{abstract}
A novel, persistent, electrochemically active perchlorinatedtriphenylmethyl (PTM) radical with a diazonium functionality has been covalently attached onto highly ordered pyrolytic graphite (HOPG) by electrografting in a single-step process. Electrochemical scanning tunneling microscopy (EC-STM) and Raman spectroscopy measurements revealed that PTM molecules have a higher tendency to covalently react at the HOPG step edges. The cross-section profiles from EC-STM images showed that there is current enhancement at the functionalized areas, which can be explained by redox mediated electron tunneling through surface-confined redox-active molecules. Cyclic voltammetry clearly demonstrated that the intrinsic properties of the organic radical are preserved upon grafting and density functional theory (DFT) calculations also reveal that the magnetic character of the PTM radical is preserved.
\end{abstract}

\section{Introduction}

In the last decades, the robust molecular modification of surfaces of different nature has been determinant for the progress of molecular electronic devices. More recently, the covalent and non-covalent chemical modification of sp2-carbon substrates and in particular graphene has awakened much interest among others, for sensing, biological, optoelectronic and catalytic applications.[1] Non-covalent modification of such substrates is usually simpler and the pristine structure of the substrate is almost unaltered. On the contrary, the covalent chemical modification is more complex but it could pave the way to solve the intrinsic drawbacks of pristine graphene, like its zero-bandgap and low solubility[2], and it leads to mechanically more robust moleculesubstrate hybrid systems.

Several synthetic methodologies have been described for achieving the covalent modification of graphite and/or graphene substrates.[3] The use of free radical addition and, in particular, through aryl diazonium salts is one of the most investigated routes.[4] The grafting of aryl radicals onto surfaces takes place under mild conditions, and it can be electrochemically induced which makes the functionalization much faster compared to other synthetic approaches. Electrochemical reduction [4a, $4 b, 5]$ of the diazonium salts using cyclic voltammetry (CV) is generally preferred, although spontaneous reduction [4f, 6] (if the substrate has reducing capability) and chemical reduction[7] have been reported in the literature as well. In some of these works, HOPG has been used as a model substrate.

Open-shell organic molecules grafted onto surfaces are gaining much attention in fields like organic radical batteries[8] supercapacitors[9] and molecular spintronics.[10] Covalent 
modification is very important in order to achieve higher stability and robustness of hybrid materials that are designed for being implemented in devices. PTM radicals are chemically and thermally persistent redox- and magnetically-active species that can be reversibly reduced/oxidized between the paramagnetic radical and the diamagnetic anion forms.[11] They can be used as switchable multifunctional materials since the magnetic, optical and electronic properties can be switched on and off with external electrochemical stimuli.[12]

In this work, we synthesize a novel PTM radical (Scheme 1), bearing a diazonium functional group for its covalent attachment onto HOPG surface through an in situ formed aryl radical. Interestingly, this implies the induced formation and temporal co-existence of a $\pi$ - and $\sigma$-radical during surface grafting (Figure 1). Previously, Cougnon et al.,[13] followed a two-step strategy in order to functionalize glassy carbon with a nitroxyl radical (TEMPO). First, the protected species (nonradical) of a stable nitroxyl group is electrochemically attached onto the surface. Then, the TEMPO radical is formed by potential cycling. TEMPO radical could not be directly grafted due to its ability to trap free radical groups. On the contrary, in this work the high stability of the PTM radical due to its sterically protected alpha carbon allows for the functionalization of the substrate in one step through the induced highly reactive aryl radicals without any evidence of side reactions between the aryl-radical and PTM-radical. To the best of our knowledge, this is the first example of an organic radical that is covalently linked to a carbon-based substrate through a direct C-C bond using diazonium chemistry in a single step surface reaction.[13-14]

\section{Results and Discussion}

Synthesis of the PTM derivative. The synthetic route depicted in Scheme 1 is followed in order to obtain the desired PTM derivative. First the triazene molecule (1) was synthesized by the reaction of 4-aminobenzaldehyde with sodium nitrite and pyrrolidine. We chose triazene as a precursor to the diazonium salt, since triazenes are highly stable to basic conditions, nucleophiles and reducing agents. However, when treated with a strong acid they yield the corresponding diazonium salts. As a result, this strategy ensures more synthetic possibilities during the synthesis. The second step was a Horner-Wadsworth-Emmons reaction performed using PTM phosphonate (2)[15] and compound 1 to form compound 3, which is a protected diazonium salt of the non-radical precursor, $\alpha \mathrm{H}$-PTM molecule. Next, PTM triazene radical ( $4 \square$ was obtained through the treatment of the $\alpha \mathrm{H}$-PTM (3) with tetrabutylammonium hydroxide (TBAOH) to generate the corresponding anion, and then oxidation of this anion to the PTM radical by silver nitrate. Then, this triazene PTM radical (4 $\square$ was converted to the diazonium salt by addition of tetrafluoroboric acid (HBF4), obtaining the desired PTM radical diazonium salt $(5 \square)$, This compound was stable for a few months when kept below $5{ }^{\circ} \mathrm{C}$. Details of this synthetic route and characterization of the molecules can be found in the Experimental Section. 


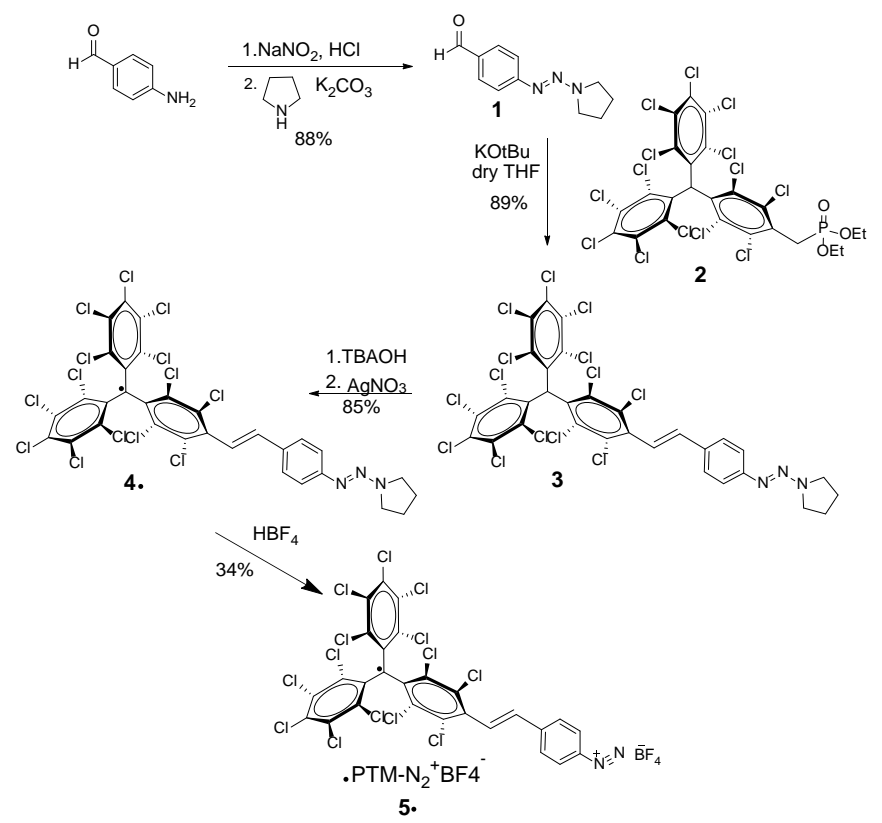

Scheme 1. Synthetic route for $\square T M-N 2 B F 4$ (5 $\square$

Modification of HOPG surface with $\square T$ TM radical diazonium salt $(5 \square$ and its characterization. In general, covalent binding of aryl radicals onto carbon-based electrodes via the electrochemical reduction of diazonium salts can be achieved through either applying consecutive potential sweeping cycles or a potential step. $[4 \mathrm{e}, 16]$ Binding of the molecules onto the substrate

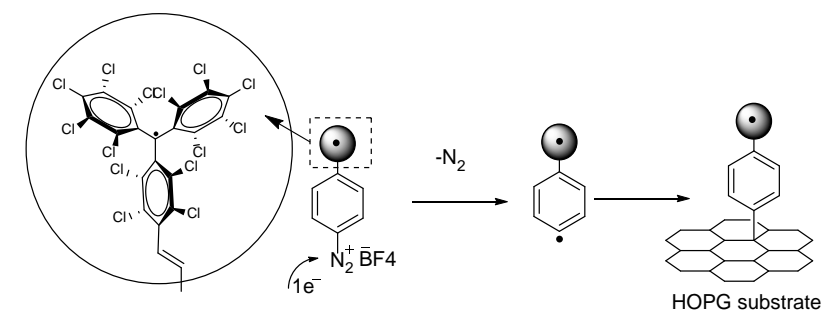

Figure 1. Schematic representation of the attachment of PTM diazonium radical on sp2-carbon substrates.

is likely a two-step process (Figure 1).[17] The first step involves the electrochemical reduction of the diazonium salt, which results in the elimination of N2 from the molecule, and generation of the intermediate aryl radicals. These reactive $\sigma$-radicals then covalently attach to the carbonbased electrode through formation of a $\mathrm{C}-\mathrm{C}$ bond, yielding surfaces functionalized with aryl groups. 
In this work, a solution $0.5 \mathrm{mM}$ of compound $5 \square$ in $0.1 \mathrm{M}$ tetrabutylammonium hexafluorophosphate in tetrahydrofuran was prepared for the electrografting experiments. As a model carbon-based surface first a glassy carbon electrode with $3 \mathrm{~mm}$ diameter was used as the working electrode in a standard three-electrode cell (see SI, S1). For the HOPG substrates a homemade electrochemical cell was utilized. In this set up, two Pt wires were used as pseudo-reference and counter electrodes. Electrochemical reduction of the diazonium salt was conducted within a potential range of $1.0 \mathrm{~V}$ (from $0.2 \mathrm{~V}$ to $-0.8 \mathrm{~V}$ ) at a scan rate of $0.2 \mathrm{~V} / \mathrm{s}$ by carrying out 3 consecutive scans. The cyclic voltammogram showed a broad irreversible cathodic peak due to the reduction of the diazonium salt into open-shell PTM substituted aryl radical at $-0.32 \mathrm{~V} v \mathrm{Pt}(\mathrm{s})$ (Figure 2a).
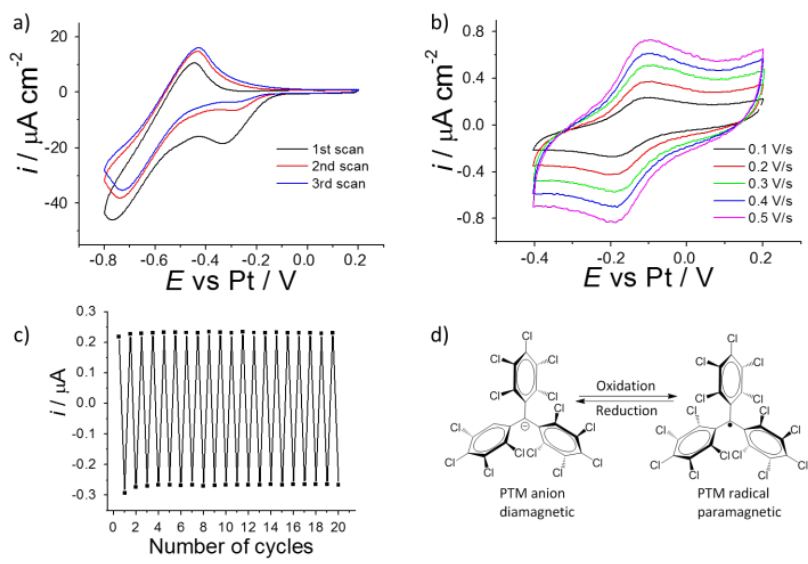

Figure 2. a) Electrografting of PTM diazonium salt 5. on HOPG substrate (3 consecutive scans, electrolyte: 0.1M TBAPF6 in tetrahydrofuran), b) Cyclic voltammogram of the electrografted PTM radical monolayer at different scan rates. Pt wires were used as pseudo-reference and counter electrode, c) Maximum current intensity during 20 cycles of the cathodic and anodic peak extracted from the PTM-monolayer CV at SR $=0.1 \mathrm{~V} / \mathrm{s}$, d) Scheme of PTM redox cycle.

The cathodic and anodic peaks at more negative potentials are ascribed to the redox process of PTM radicals diffusing from the bulk of the solution to the surface. The intensity of the peak due to diazonium reduction decreased gradually in the subsequent CV scans, which was attributed to the passivation of the reactive regions of the HOPG substrate as the reaction proceeds.[18] We do not find the formation of thick polyaryl layers plausible, due to the steric hindrance provided by the bulky PTM moieties.[19] To eliminate any unreacted material, the modified surface was vigorously rinsed with solvents before any further characterization. The CVs of the modified HOPG after electrografting (Figure $2 \mathrm{~b}$ ) displayed one pair of reversible redox peaks (mid-peak potential Emid= $-0.15 \mathrm{~V}$ vs Pt(s)) that corresponded to the redox process between the PTM radical and the PTM anion (Figure $2 \mathrm{~d}$ ). The peak intensities increased linearly with increasing scan rate as expected for surface-confined electro-active species. Full-width peak, $\Delta$ Efwhm, which is a parameter that is used to indicate the interaction between the redox centers, was found to be $165 \mathrm{mV}$ at $0.2 \mathrm{~V} / \mathrm{s}$ scan rate. In an ideal situation where there are no interactions between the redox centers on the 
substrate $\Delta$ Efwhm would be $90.6 \mathrm{mV}$.[20] We attribute the deviation from this value to some degree of repulsive electrostatic interaction between the PTM moieties.

The surface coverage (Г) was calculated to be $4.5 \times 10-12 \mathrm{~mol} / \mathrm{cm} 2$ (details of calculation see SI, S1). This value is much lower than what is theoretically estimated for a flat homogeneous surface $(1.85 \times 10-10 \mathrm{~mol} / \mathrm{cm} 2$, see SI, Figure S2c) but it is in accordance with the EC-STM experiments shown below. The coverage was ten times higher when the same experiments were performed using glassy carbon electrode. Thus, the low coverage found on HOPG can be attributed to the lower reactivity of this substrate due to the lower amount of defect sites on the basal plane. In order to check the stability of the formed monolayer and the reversibility of the redox process, multiple redox cycles were applied. Upon 20 consecutive scans at $0.1 \mathrm{~V} / \mathrm{s}$ the intensity of the peaks stayed nearly constant (Figure 2c). Remarkably, as it was previously observed for PTM radicals grafted on gold[21] and indium-tin oxide[22], PTM-modified HOPG surfaces also have the potential to perform as an electrochemical switch showing two distinguishable states, anionPTM-HOPG and radicalPTM-HOPG.

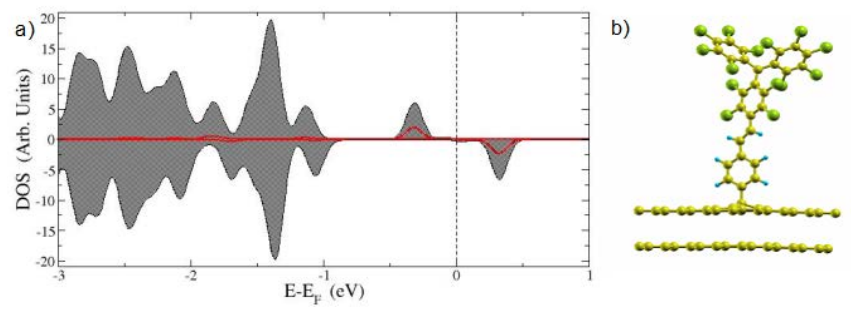

Figure 3. a) DOS of the PTM molecule (grey shaded area) and DOS projected over the PTM trivalent central carbon atom coordinated by the chlorinated phenyl rings (red line). Positive/Negative values of the DOS are for up/down spin. The SOMO and SUMO, which are located at an energy around the Fermi level, are clearly distinguishable. Note that the SOMOSUMO gap may be underestimated by DFT. b) Optimized structure for the PTM on graphite.

Density functional theory (DFT) calculations supports that the PTM moiety preserves its radical state upon grafting on the HOPG surface. The well-separated singly occupied and singly unoccupied molecular orbitals (SOMO and SUMO) of the PTM radical adsorbed on a terrace of HOPG are clearly visible in the density of states (DOS) (Figure 3). Figure $3 b$ shows the optimized structure for the PTM on graphite. Here the PTM molecule was assumed to be adsorbed on a terrace but it was observed that the SOMO and SUMO DOS depend weakly on whether the PTM binds at the terrace or at an edge. Furthermore, the net charge of central PTM carbon atom coordinated to the chlorinated phenyl rings has been computed through the Hirshfeld populations and is equal to $-0.01 \mathrm{e}$; a very small value that compares with that for the gas phase bare PTM, 0.0057 e. Similar results were found also for the molecule bonded to the edges (see SI, Figure S2a).

Further, modified HOPG surfaces were examined via X-ray photoelectron spectroscopy (XPS), Raman spectroscopy and EC-STM. Figure 4 shows the high-resolution XPS spectra for $\mathrm{C} 1 \mathrm{~s}(\mathrm{a}), \mathrm{Cl} 2 \mathrm{p}$ (b) and N1s (c). The C1s spectrum displayed an intense narrow peak at a binding energy of 284.5 eV confirming high sp2 carbon content. This peak was accompanied by two smaller peaks at 285.0 
$\mathrm{eV}$ (C sp3) and $286.2 \mathrm{eV}(\mathrm{C}-\mathrm{Cl})$, and a $\pi-\pi^{*}$ shake up satellite peak at $291.0 \mathrm{eV}$. The $\mathrm{C}-\mathrm{Cl}$ peak was experimentally not observed for the bare HOPG (inset Figure 4a). The origin of the observed features can be rationalized by simulations carried out using DFT. Calculations showed that the $\mathrm{C} 1 \mathrm{~s}$ for the HOPG atom covalently bonded to the PTM molecule was shifted toward higher binding energies by $0.69 \mathrm{eV}$ with respect to the $\mathrm{C} 1 \mathrm{~s}$ for the pristine HOPG surface (see SI, Figure S2b). On the other hand, a larger shift equal to $1.28 \mathrm{eV}$ was found for the $\mathrm{C} 1 \mathrm{~s}$ of the PTM C-Cl. These shifted states could be therefore identified with the two small peaks observed experimentally. The computed core level shifts account significantly well for the XPS spectrum of the modified HOPG thus confirming the increased sp3 character of the surface and the presence of the PTM molecule.

(a)

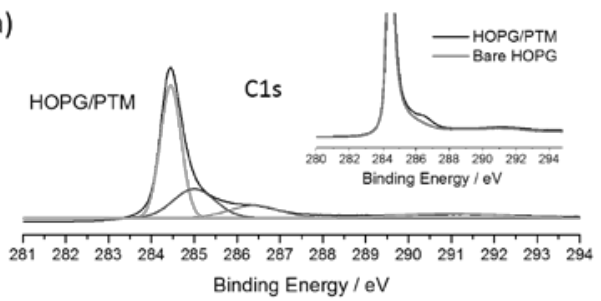

(b)

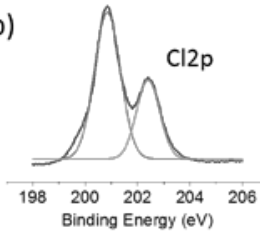

(c)

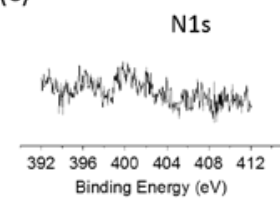

Figure 4. High resolution $\mathrm{C} 1 \mathrm{~s}(\mathrm{a}), \mathrm{Cl} 2 \mathrm{p}(\mathrm{b})$ and $\mathrm{N} 1 \mathrm{~s}$ (c) regions of the XPS spectrum of the HOPG substrate modified with PTM radical. C1s spectrum of bare HOPG is also shown (inset 4a) in comparison to modified HOPG.
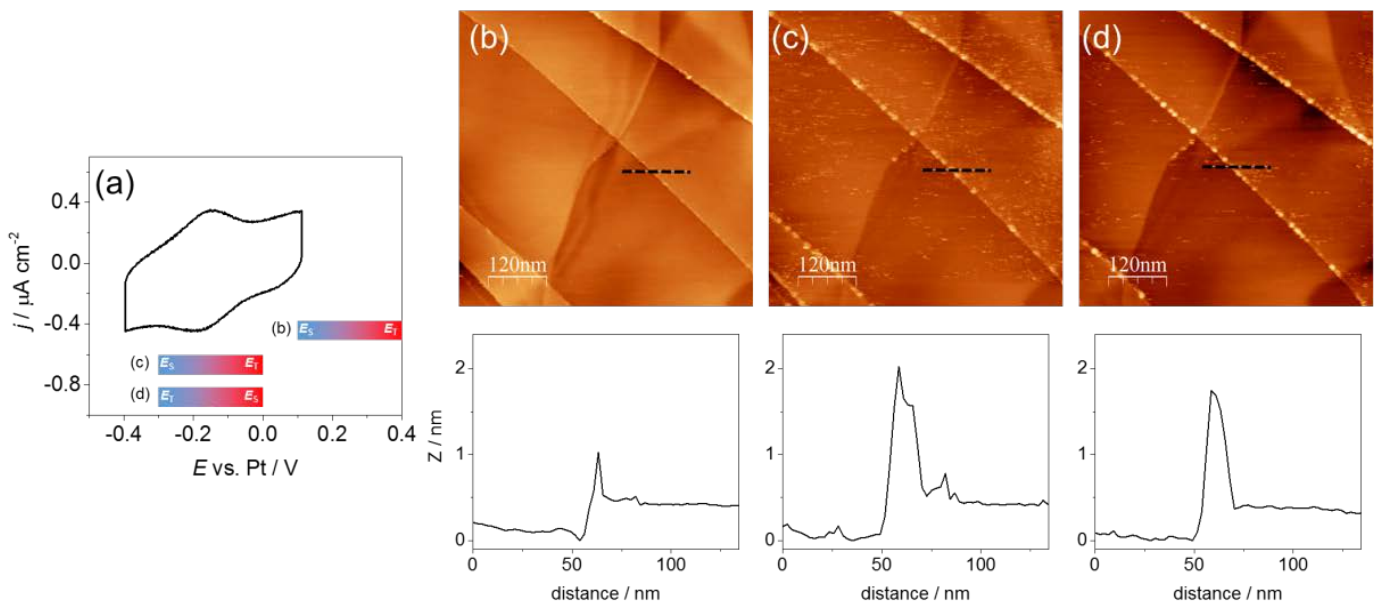
Figure 5. (a) Voltammogram of the modified HOPG substrate. (b-d) In situ STM images of PTM radical functionalized HOPG surfaces and respective cross-sections, in [SEt3]-[Tf2N]+ Et3N (15:1 $\mathrm{V} / \mathrm{V}$ ) at (b) $\mathrm{ES}=0.10 \mathrm{~V}, \mathrm{ET}=0.40 \mathrm{~V}$, (c) $\mathrm{ES}=-0.30 \mathrm{~V}, \mathrm{ET}=0.00 \mathrm{~V}$, and (d) $\mathrm{ES}=0.00 \mathrm{~V}, \mathrm{ET}=-0.30 \mathrm{~V}$ (Vs $\mathrm{Pt}(\mathrm{s}))$. The bias voltage, Ebias, was $(\mathrm{b}, \mathrm{c}) 0.30 \mathrm{~V}$ and $(\mathrm{d})-0.30 \mathrm{~V}$; ISP $=0.1 \mathrm{nA}$ for all images shown here.

This is further demonstrated by the analysis of the $\mathrm{Cl} 2 \mathrm{p}$ experimental spectrum. The peak was deconvoluted into two peaks at $202.5 \mathrm{eV}$ and $200.9 \mathrm{eV}$ that correspond to $\mathrm{Cl} 2 \mathrm{p} 1 / 2$ and $\mathrm{Cl} 2 \mathrm{p} 3 / 2$ respectively, at a 1:2 intensity ratio and with an energy difference of $1.6 \mathrm{eV}$ as expected, exhibiting the presence of chlorine atoms from the PTM moieties on the surface. Remarkably, the absence of peaks in the N1s region of the spectrum, which would correspond to the presence of $\mathrm{N}-\mathrm{H}(400.0$ $\mathrm{eV}$ ) and diazo bonds $(402.0 \mathrm{eV})$ confirmed that there were no unreacted diazonium molecules on the substrate.[4f]

Scanning Tunneling Microscopy on HOPG Surfaces. For EC-STM experiments the grafting of $5 \square$ was performed directly in STM cell under argon atmosphere (see SI, Figure S3a-b). Then, the modified HOPG electrode and the cell were thoroughly rinsed with different solvents, and an electrolyte solution was added to perform voltammetric and STM measurements. Triethylsulfonium bis(trifluoromethylsulfonyl) imide was dried with molecular sieves, and used as electrolyte solution with an additive of triethylamine for EC-STM. The ionic liquid (IL) was used for its lower volatility, which was necessary while performing long experiments in the EC-STM cell. Et3N molecules were added to protect PTM anionic species from uncontrollable protonation during the cyclic voltammetry measurements.

The cyclic voltammogram of $5 \square$ HOPG as recorded in the CV set-up is shown in Figure $5 a$. Reversible peaks at around $-0.16 \mathrm{~V}$ vs Pt(s) are due to the redox process between the PTM radical and anion, with a peak-to-peak separation $(\Delta \mathrm{E})$ of $25 \mathrm{mV}$. Under these conditions surface coverage on HOPG was calculated to be $\sim 6 \times 10-12 \mathrm{~mol} / \mathrm{cm} 2$, in accordance with the previous experiments described above. Figures $5 b-d$ show in situ STM images of the same area of $5 \square$ HOPG substrate recorded at different potentials of the substrate ES and a gold tip ET. Cross-section profiles taken along black dashed lines are given below the images. The STM images show a clear tendency of the radical PTM molecules to react at the step edges of the HOPG compared to the terraces. As an example, the cross section profile in Figure $5 b$ reveals increased height at the step edges of the graphite confirming the covalent attachment of molecules at these sites. After several STM scans the molecules remain unaltered pointing to a strong molecule-HOPG bond. The apparent height (z) of step edges increased significantly (from 0.6 to $1.5 \mathrm{~nm}$ ) along with brightening of these areas (Figures $5 b-d$ ) when ES and ET were set before and after the redox peaks. The choice of electrode potentials with respect to the redox signal is illustrated in Figure 5a. Moreover, we observed the increasing apparent height of step edges at both positive and negative bias voltages (Figures $5 \mathrm{c}$ and $5 \mathrm{~d}$ ). Since the images were recorded in a constant-current mode, the increasing apparent height of the surface features (or their STM contrast) reflects the amplified conductivity (or 
enhanced tunnelling current). Notice that all images were recorded at the same absolute value of bias voltage $(0.30 \mathrm{~V})$, which means that the enhancement of tunnelling current was not related to change of bias voltage. It was also not related to the intercalation of IL ions into the steps, as we did not observe any change in STM images upon ES and ET shift, when a reference experiment with bare HOPG substrate was performed (see SI, Figure S3c). The observed current enhancement at the step edges of $5 \square$ HOPG can be explained by redox-mediated electron tunnelling (RMET) through surface-confined redox-active molecules.[23] RMET was experimentally observed by STM (and/or tunnelling spectroscopy) e.g. for the adlayers on gold terminated by redox-active moieties such as viologens, ferrocene-, and transition metal complexes.[23a-c, 24] Thus, the in situ STM measurements confirm the strong binding of redox-active PTM radicals to HOPG step edges. However, we did not obtain a solid evidence for the attachment of $5 \square$ to HOPG terraces. The higher reactivity of the edges when compared to the basal plane of the HOPG has been previously reported.[19, 25]

Raman spectroscopy was used in order to assess the surface characteristics of the modified HOPG substrates. Different areas of the substrate were investigated: some areas were flat, but some contained many steps (step-bunched regions). Figure 6 shows representative Raman spectra from these two types of regions on the HOPG substrate.

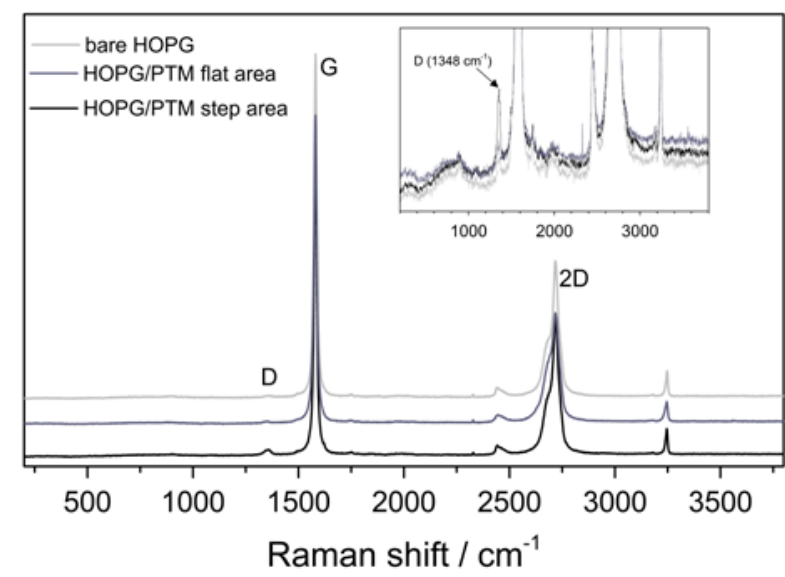

Figure 6. Representative Raman spectra of HOPG-PTM (covalently grafted) and bare HOPG. Spectra were obtained for a flat area of HOPG and for a step-bunched area.

The spectrum for the unmodified flat HOPG area displayed a strong and sharp $\mathrm{G}$ band at $1580 \mathrm{~cm}$ 1 and a smaller and broader $2 \mathrm{D}$ band at $2717 \mathrm{~cm}-1$. D band that is a fingerprint for sp3 defects at a sp2-carbon substrate[26] was almost absent for the unmodified HOPG indicating nearly defectfree surface (intensity ratio ID/IG $=2.5 \times 10-3$ ). Similarly, the $2 \mathrm{D}$ band at $2714 \mathrm{~cm}-1$ and the sharp $\mathrm{G}$ band at $1579 \mathrm{~cm}-1$ were observed for the modified substrates. On a flat area, where the density

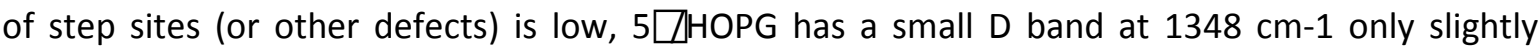
higher than that for bare HOPG (ID/IG $=5.8 \times 10-3)$. But, for step-bunched regions, the $D$ band is much more pronounced, when HOPG surface was modified by $5 \square$ ID/IG is $3.5 \times 10-3$ and $14.2 \times$ 10-3 for unmodified and modified HOPG, respectively). These results support our proposal that $5 \square$ 
modifies preferentially defects of HOPG surface and the sites adjacent to the defects (due to formation of adjacent sp3 nodes).

\section{Conclusions}

We have presented the synthesis of a novel PTM organic radical with a diazonium moiety for successful covalent functionalization of carbon-based surfaces by electrografting. The aryl radical formation through reduction of the diazonium group did not alter the redox properties of the functional radical PTM moiety. The study of the electrolyte/radical-HOPG interface by EC-STM measurements illustrates the high stability of the grafted functional species. Also, it shows that HOPG surfaces were mainly functionalized at the step-edges and that the grafted organic radicals could serve as redox-mediators for electron tunnelling and tune tunnelling current upon adjustment of the STM tip and substrate potentials. Furthermore, the electrochemical experiments demonstrated the potential of this organic hybrid material to behave as molecular switches and opening the possibility to be explored as memories.

\section{Experimental Section}

\section{Synthesis}

Synthesis of compound 1. A solution of 4-aminobenzaldehyde (170 mg, $1.4 \mathrm{mmol})$ in concentrated $\mathrm{HCl}(0.55 \mathrm{ml})$ was cooled in an ice bath to $0 \square$. To this solution, a mixture of sodium nitrite (102 $\mathrm{mg}, 1.5 \mathrm{mmol})$ in cold water $(5.6 \mathrm{ml})$ was added dropwise. Resulting diazonium salt was stirred at 0 $\square$ for $30 \mathrm{~min}$ and then added at once to a solution of pyrrolidine $(0.23 \mathrm{ml}, 2.8 \mathrm{mmol})$ and potassium carbonate $(970 \mathrm{mg}, 7 \mathrm{mmol})$ in $1: 2$ acetonitrile:water $(2.3 \mathrm{ml}: 4.6 \mathrm{ml})$. The reaction mixture was allowed to warm to room temperature and stirred for $1 \mathrm{~h}$. Then, the aqueous phase was extracted with dichloromethane $(3 \times 50 \mathrm{ml})$. Combined organic layers were washed with brine twice, dried over magnesium sulfate, filtered and concentrated under reduced pressure. The crude product was purified by chromatography (silica gel with ethyl acetate: hexanes, 2:10) to yield the desired triazene product $(250 \mathrm{mg}, 88 \%) .1 \mathrm{H} \mathrm{NMR}(\mathrm{CDCl} 3) \delta(\mathrm{ppm}) 9.92(\mathrm{~s}, 1 \mathrm{H}), 7.82(\mathrm{~d}, \mathrm{~J}=7.5 \mathrm{~Hz}$, $2 \mathrm{H}), 7.51$ (d, J = 7.5Hz, 2H), 3.95 (br s, 2H), 3.70 (br s, 2H), 2.05 (br s. 4H). 13C NMR (CDCl3) $\delta(p p m)$ 191.5, 156.4, 133.2, 131.0, 120.7, 51.4, 46.7, 29.7, 23.7. IR (ATR): 2957, 2928, 2873, 2714, 1691, $1591,1391,1304,1205,1134,1107,847,826 \mathrm{~cm}-1$

Synthesis of compound 3. Under dry conditions, $96 \mathrm{mg}(0.85 \mathrm{mmol})$ of potassium-tert-butoxide was added to a solution of PTM phosphonate salt $(460 \mathrm{mg}, 0.52 \mathrm{mmol}$ ) in $6 \mathrm{ml}$ of dry THF at -78 व. This mixture was stirred for $30 \mathrm{~min}$, and then $136 \mathrm{mg}(0.68 \mathrm{mmol})$ of aldehyde 1 dissolved in 3 $\mathrm{ml}$ of dry THF was added. The reaction mixture was allowed to warm to room temperature and stirred under inert atmosphere around $15 \mathrm{~h}$. Then, $1 \mathrm{~N} \mathrm{HCl}(4 \mathrm{ml})$ was added until the color of the solution is light brown. Extraction with dichloromethane was done $(3 \times 30 \mathrm{ml})$. Combined organic layers were washed with brine $(2 \times 30 \mathrm{ml})$, dried over magnesium sulfate, filtered and the solvent was evaporated under reduced pressure. The crude product was purified by chromatography (silica gel with ethyl acetate: hexanes, 1:10) to yield the desired product (430 mg, 89\%). LC-MS (positive mode) $(\mathrm{C} 31 \mathrm{H} 15 \mathrm{Cl} 14 \mathrm{N3}, \mathrm{M}=925.81) ;(\mathrm{m} / \mathrm{z})[\mathrm{M}]=925.6,[\mathrm{M}-97]=828.5 .1 \mathrm{H} \mathrm{NMR}(\mathrm{CDCl} 3)$ 
$\delta(\mathrm{ppm}) 7.49(\mathrm{~d}, \mathrm{~J}=8.4 \mathrm{~Hz}, 2 \mathrm{H}), 7.43(\mathrm{~d}, \mathrm{~J}=8.4 \mathrm{~Hz}, 2 \mathrm{H}), 7.07(\mathrm{~d}, \mathrm{~J}=16.2 \mathrm{~Hz}, 1 \mathrm{H}), 7.00(\mathrm{~d}, 2 \mathrm{H}), 6.97(\mathrm{~s}$, $1 \mathrm{H}), 3.81(\mathrm{br} \mathrm{s}, 4 \mathrm{H}), 2.02(\mathrm{~s}, 4 \mathrm{H})$.

Synthesis of compound $4 \cdot 180 \mathrm{mg}(0.19 \mathrm{mmol})$ of 3 was dissolved in $6 \mathrm{ml}$ of dry THF. To this solution a 140 Dof tetrabutylammonium hydroxide solution in $\mathrm{H} 2 \mathrm{O}$ (54-56 \%) was added. The reaction mixture was stirred for 30min and then $70 \mathrm{mg}(0.41 \mathrm{mmol})$ of AgNO3 was added. After the formation of the products were confirmed by UV-Vis spectroscopy the reaction mixture was poured over a column packed with silica gel and filtered. The residue at the top of the column was further washed with DCM, and then combined filtrates were concentrated under reduced pressure. The crude product was purified by chromatography (silica gel with ethyl acetate: hexanes, 2:10) to yield the desired product (150mg, 85\%). LC-MS (positive mode) (C31H14Cl14N3, $\mathrm{M}=$ 924.81); (m/z) [M] = 924.6; [M-67] = 857.6; [M-97] = 827.5. UV-Vis (THF) $\lambda \max (\square): 236 \mathrm{~nm}$ (54770), $369 \mathrm{~nm}$ (36769), $388 \mathrm{~nm}$ (46311), $505 \mathrm{~nm}$ (8287), $695 \mathrm{~nm}$ (2546). Electrochemistry [ $\mathrm{NaClO} 4$ in $\mathrm{CH} 3 \mathrm{CN}, \mathrm{Ag} / \mathrm{AgCl}]: \mathrm{E} 1 / 2$ (radical/anion) $=-0.11 \mathrm{~V}, \mathrm{E} 1 / 2$ (radical/cation) $=0.96 \mathrm{~V} . \mathrm{EPR} \mathrm{g}=$ $2.0032 \mathrm{aH}=1.99 \mathrm{G}, \mathrm{a} 13 \mathrm{C} \alpha=29.9 \mathrm{G}, \mathrm{a} 13 \mathrm{Carom}=12.0 \mathrm{G}$

Synthesis of compound $5.63 \mathrm{mg}(0.07 \mathrm{mmol})$ of PTM triazene radical 4 was dissolved in $2 \mathrm{ml}$ of dry THF. This solution was cooled with an ice bath to around $0-5 \square$, and then $1.0 \mathrm{ml}$ of HBF4 solution in $\mathrm{H} 2 \mathrm{O}$ ( $48 \mathrm{wt} \%$ ) was added dropwise. The reaction mixture was stirred for about $10 \mathrm{~min}$. After confirming the formation of products by UV-Vis spectroscopy the reaction was terminated by the addition of diethyl ether $(60 \mathrm{ml})$. The formation of red small crystals was observed within a few minutes. These crystals were washed with cold ether multiple times, and then dried under reduced pressure to yield the desired compound (23 mg, 34\%). IR-ATR umax (cm-1) 3101, 2259, $1579,1336,1072,815,733$. EPR $g=2.0024, a H=2.0 \mathrm{G}, \mathrm{a} 13 \mathrm{C} \alpha=29.7 \mathrm{G}$, a13Carom $=12.1 \mathrm{G}$, UV-Vis (THF) $\lambda \max (\square] 234 \mathrm{~nm}$ (66302), $333 \mathrm{~nm}$ (24264), $385 \mathrm{~nm}$ (24917). Electrochemistry [NaClO4 in $\mathrm{CH} 3 \mathrm{CN}, \mathrm{Ag} / \mathrm{AgCl}$ : $\mathrm{E} 1 / 2$ (radical/anion) $=-0.16 \mathrm{~V}$, Ediazonium reduction $=0.02 \mathrm{~V}$

\section{Theoretical calculations}

Density Functional Theory (DFT) calculations were carried out with the all-electron code FHIAIMS[27] The Perdew-Burke-Ernzerhof (PBE) generalized gradient approximation[28] to the exchange-correlation density functional was used together with numerical atom centered basis, where the free-atom-like radial orbitals are improved by adding further radial functions grouped in the tier 2 set. This corresponds to the pre-constructed "tight" default setting. A supercell containing the molecule and a slab composed of three graphene layers was used to model the system. The geometry optimization was performed until the ionic forces were smaller than 0.01 eV/Ang while fixing the bottom graphene layer of the slab. The inclusion of van der Waals corrections[29] is required to describe the HOPG interlayer binding[30], but has a negligible effect on the final results for the molecule absorption geometry and electronic structure. A $10 \times 10 \times 1 \mathrm{k}-$ point grid was chosen.

The Kohn-Sham states were considered good approximations to the real quasi-particle states of the system, although this may result in the underestimation of the gap between the singly occupied and singly unoccupied molecular orbitals (SOMO-SUMO). A Gaussian broadening of 0.05 eV was applied to the density of states (DOS) in order to obtain smooth peaks. The atom-projected 
DOS was based on the Mulliken population analysis. Complementary results for the net charge of each atom were obtained by looking the Hirshfeld atomic populations.[31]

\section{Acknowledgements}

We acknowledge Guillaume Sauthier from Catalan Institute of Nanoscience and Nanotechnology (ICN2) for XPS measurements. This work was supported by ACMOL (GA no. 618082), the Swiss National Science Foundation (Grant No. 200020-144471), ERC StG 2012-306826 e-GAMES, the Networking Research Center of Bioengineering, Biomaterials and Nanomedicine (CIBER-BBN), the DGI (Spain) with project BE-WELL CTQ2013-40480-R and the Generalitat de Catalunya with project 2014-SGR-17. N.C acknowledges the RyC program. The authors also acknowledge financial support from the Spanish Ministry of Economy and Competitiveness, through the "Severo Ochoa" Programme for Centres of Excellence in R\&D (SEV- 2015-0496)

Keywords: organic radicals • HOPG • diazonium chemistry • electrochemistry • surface modification

[a] Dr. G. Seber, Prof. J. Veciana, Dr. M. Mas-Torrent, Prof. C. Rovira, Dr. N. Crivillers

Institut de Ciència de Materials de Barcelona (CSIC) and CIBER-BBN, Campus de la UAB, 08193, Bellaterra, Spain.

E-mail: ncrivillers@icmab.es

[b] Dr. A. Rudnev

Department of Chemistry and Biochemistry, University of Bern, Freistrasse 3, CH-3012 Bern, Switzerland. A.N. Frumkin Institute of Physical Chemistry and Electrochemistry RAS, Leninskii pr.31, Moscow, 119991, Russia.

E-mail: rudnev@dcb.unibe.ch

[c] Dr. A Droghetti

Nano-Bio Spectroscopy Group and European Theoretical Spectroscopy Facility (ETSF), Universidad del País Vasco, Av. Tolosa 72, 20018, San Sebastian, Spain.

[d] Dr. I. Rungger

Materials Division, National Physical Laboratory, Teddington, TW11 OLW, United Kingdom 
[1] a)V. Georgakilas, M. Otyepka, A. B. Bourlinos, V. Chandra, N. Kim, K. C. Kemp, P. Hobza, R. Zboril, K. S. Kim, Chem. Rev. 2012, 112, 6156-6214; b)J. Liu, J. Tang, J. J. Gooding, J. Mat. Chem. 2012, 22, 12435-12452.

[2] K. S. Novoselov, A. K. Geim, S. V. Morozov, D. Jiang, Y. Zhang, S. V. Dubonos, I. V. Grigorieva, A. A. Firsov, Science 2004, 306, 666-669.

[3] C. K. Chua, M. Pumera, Chem. Soc. Rev. 2013, 42, 3222-3233.

[4] a)M. Delamar, R. Hitmi, J. Pinson, J.-M. Savéant, J. Am. Chem. Soc. 1992, 114, 5883-5884; b)P. Allongue, M. Delamar, B. Desbat, O. Fagebaume, R. Hitmi, J. Pinson, J.-M. Savéant, J. Am. Chem. Soc. 1997, 119, 201-207; c)Y. Liu, R. L. McCreery, J. Am. Chem. Soc. 1995, 117, 1125411259; d)A. J. Downard, Electroanalysis 2000, 12, 1085-1096; e)M. Bernard, A. Chaussé, E. CabetDeliry, M. M. Chehimi, J. Pinson, F. Podvorica, C. Vautrin-UI, Chem. Mater. 2003, 15, 3450-3462; f)A. Adenier, E. Cabet-Deliry, A. Chaussé, S. Griveau, F. Mercier, J. Pinson, C. Vautrin-UI, Chem. Mater. 2005, 17, 491-501; g)J. K. Kariuki, M. T. McDermott, Langmuir 2001, 17, 5947-5951; h)K. Boukerma, M. M. Chehimi, J. Pinson, C. Blomfield, Langmuir 2003, 19, 6333-6335.

[5] A. Adenier, M. Bernard, M. M. Chehimi, E. Cabet-Deliry, B. Desbat, O. Fagebaume, J. Pinson, F. Podvorica, J. Am. Chem. Soc. 2001, 123, 4541-4549.

[6] a)J. Lehr, B. E. Williamson, B. S. Flavel, A. J. Downard, Langmuir 2009, 25, 13503-13509; b)F. Le Floch, J.-P. Simonato, G. Bidan, Electrochim. Acta 2009, 54, 3078-3085; c)A. Adenier, N. Barré, E. Cabet-Deliry, A. Chaussé, S. Griveau, F. Mercier, J. Pinson, C. Vautrin-UI, Surf. Sci. 2006, 600, 4801-4812; d)J. L. Bahr, J. M. Tour, Chem. Mater. 2001, 13, 3823-3824.

[7] a)G. G. Wildgoose, H. C. Leventis, I. J. Davies, A. Crossley, N. S. Lawrence, L. Jiang, T. G. J. Jones, R. G. Compton, J. Mater. Chem. 2005, 15, 2375-2382; b)C. G. Heald, G. G. Wildgoose, L. Jiang, T. G. Jones, R. G. Compton, Chem. Phys. Chem. 2004, 5, 1794-1799; c)A. Mesnage, S. p. Esnouf, P. Jégou, G. Deniau, S. Palacin, Chem. Mater. 2010, 22, 6229-6239.

[8] a)T. Janoschka, M. D. Hager, U. S. Schubert, Adv. Mater. 2012, 24, 6397-6409; b)A. Y. Tesio, D. Blasi, M. Olivares-Marin, I. Ratera, D. Tonti, J. Veciana, Chem. Commun. 2015, 51, 17623-17626; c)A. Aqil, A. Vlad, M.-L. Piedboeuf, M. Aqil, N. Job, S. Melinte, C. Detrembleur, C. Jerome, Chem. Commun. 2015, 51, 9301-9304.

[9] A. Vlad, N. Singh, S. Melinte, J. F. Gohy, P. M. Ajayan, Scientific Reports 2016, DOI: 10.1038/srep22194.

[10] a)R. Frisenda, R. Gaudenzi, C. Franco, M. Mas-Torrent, C. Rovira, J. Veciana, I. Alcon, S. T. Bromley, E. Burzurí, H. S. J. van der Zant, Nano Lett. 2015, 15, 3109-3114; b)M. Mas-Torrent, N. Crivillers, V. Mugnaini, I. Ratera, C. Rovira, J. Veciana, J. Mater. Chem. 2009, 19, 1691-1695; c)J. Liu, H. Isshiki, K. Katoh, T. Morita, B. K. Breedlove, M. Yamashita, T. Komeda, J. Am. Chem. Soc. 2013, $135,651-658$.

[11] M. Ballester, J. Riera-Figueras, J. Castaner, C. Badfa, J. M. Monso, J. Am. Chem. Soc. 1971, 93, 2215-2225. 
[12] a)C. Sporer, I. Ratera, D. Ruiz-Molina, Y. Zhao, J. Vidal-Gancedo, K. Wurst, P. Jaitner, K. Clays, A. Persoons, C. Rovira, J. Veciana, Angew. Chem. Int. Ed. 2004, 43, 5266-5268; b)C. Simão, M. Mas-Torrent, J. Veciana, C. Rovira, Nano Lett. 2011, 11, 4382-4385.

[13] C. Cougnon, S. Boisard, O. Cador, M. Dias, E. Levillain, T. Breton, Chem. Commun. 2013, 49, 4555-4557.

[14] a)C. Bosch-Navarro, F. Busolo, E. Coronado, Y. Duan, C. Martí-Gastaldo, H. Prima-Garcia, J. Mater. Chem. C 2013, 1, 4590-4598; b)Z. Du, W. Ai, L. Xie, W. Huang, J. Mater. Chem. A 2014, 2, 9164-9168.

[15] C. Rovira, D. Ruiz-Molina, O. Elsner, J. Vidal-Gancedo, J. Bonvoisin, L. J., J. Veciana, Chem. Eur. J. 2001, 7, 240-250.

[16] A. J. Downard, Langmuir 2000, 16, 9680-9682.

[17] C. P. Andrieux, J. Pinson, J. Am. Chem. Soc. 2003, 125,14801-14806.

[18] a)J. Pinson, F. Podvorica, Chem. Soc. Rev. 2005, 34, 429-439; b)J. Pinson, in Aryl Diazonium Salts, Wiley-VCH Verlag GmbH \& Co. KGaA, 2012, pp. 1-35.

[19] J. Greenwood, T. H. Phan, Y. Fujita, Z. Li, O. Ivasenko, W. Vanderlinden, H. Van Gorp, W. Frederickx, G. Lu, K. Tahara, Y. Tobe, H. Uji-i, S. F. L. Mertens, S. De Feyter, ACS Nano 2015, 9, 5520-5535.

[20] A. J. Bard, L. R. Faulkner, Electrochemical Methods: Fundamentals and Applications, John Wiley \& Sons, New York, 1980 pp.522.

[21] N. Crivillers, M. Mas-Torrent, J. Vidal-Gancedo, J. Veciana, C. Rovira, J. Am. Chem. Soc. $2008,130,5499-5506$.

[22] C. Simão, M. Mas-Torrent, N. Crivillers, V. Lloveras, J. M. Artés, P. Gorostiza, J. Veciana, C. Rovira, Nat. Chem. 2011, 3, 359-364.

[23] a)Z. Li, Y. Liu, S. F. L. Mertens, I. V. Pobelov, T. Wandlowski, J. Am. Chem. Soc. 2010, 132, 8187-8193; b)I. V. Pobelov, Z. Li, T. Wandlowski, J. Am. Chem. Soc. 2008, 130, 16045-16054; c)A. V. Rudnev, I. V. Pobelov, T. Wandlowski, J. Electroanal. Chem. 2011, 660, 302-308; d)A. M. Kuznetsov, J. Ulstrup, J. Phys. Chem. A 2000, 104, 11531-11540; e)J. Zhang, A. M. Kuznetsov, I. G. Medvedev, Q. Chi, T. Albrecht, P. S. Jensen, J. Ulstrup, Chem. Rev. 2008, 108, 2737-2791.

[24] a)A. V. Rudnev, U. Zhumaev, T. Utsunomiya, C. Fan, Y. Yokota, K.-i. Fukui, T. Wandlowski, Electrochim. Acta 2013, 107, 33-44; b)Y. Yokota, K.-i. Fukui, T. Enoki, M. Hara, J. Phys. Chem. C 2007, 111, 7561-7564; c)T. Albrecht, A. Guckian, A. M. Kuznetsov, J. G. Vos, J. Ulstrup, J. Am. Chem. Soc. 2006, 128, 17132-17138.

[25] a)J. K. Kariuki, M. T. McDermott, Langmuir 1999, 15, 6534-6540; b)H. Ma, L. Lee, P. A. Brooksby, S. A. Brown, S. J. Fraser, K. C. Gordon, Y. R. Leroux, P. Hapiot, A. J. Downard, J. Phys. Chem. C 2014, 118, 5820-5826. 
[26] a)S. Niyogi, E. Bekyarova, M. E. Itkis, H. Zhang, K. Shepperd, J. Hicks, M. Sprinkle, C. Berger, C. N. Lau, W. A. deHeer, E. H. Conrad, R. C. Haddon, Nano Lett. 2010, 10, 4061-4066; b)Q. H. Wang, Z. Jin, K. K. Kim, A. J. Hilmer, G. L. Paulus, C. J. Shih, M. H. Ham, J. D. Sanchez-Yamagishi, K. Watanabe, T. Taniguchi, J. Kong, P. Jarillo-Herrero, M. S. Strano, Nat. Chem. 2012, 4, 724-732.

[27] V. Blum, R. Gehrke, F. Hanke, P. Havu, V. Havu, X. Ren, K. Reuter, M. Scheffler, Comput. Phys. Commun. 2009, 180, 2175-2196.

[28] a) J.P. Perdew, K. Burke, M. Ernzerhof, Phys. Rev. Lett. 1996, 77, 3865-3868 and Phys. Rev. Lett. 1997, 78, 1396.

[29] A. Tkatchenko, M. Scheffler, Phys, Rev. Lett. 2009, 102, 073005

[30] V.V. Gobre, A. Tkatchenko, Nat. Commun. 2013, 4, DOI:10.1038/ncomms3341

[31] F. L. Hirshfeld, Theor. Chim. Acta. 1977, 44, 129-138. 